\title{
Uso de ferramentas de abordagem familiar como balizadoras na construção do cuidado à família vulnerável na atenção básica
}

\author{
The use of family approach tools as beacons in the construction of care for vulnerable \\ families in primary care
}
El uso de herramientas de enfoque familiar como faros en la construcción de la atención a familias vulnerables en la atención primaria

Élida Chaves de Carvalho Lima ${ }^{1 *}$, Eliana Brugin Serra ${ }^{4}$, Janielle Ferreira de Brito Lima ${ }^{2}$, Geysa Santos Góis Lopes ${ }^{3}$, Isaura Leticia Tavares Palmeira Rolim¹.

\section{RESUMO}

Objetivo: Relatar a experiência da equipe da estratégia de saúde da família no uso de ferramentas de abordagem familiar para estratificação do risco de vulnerabilidade. Relato de experiência: A seleção da família ocorreu no mês de outubro de 2020 e, a partir deste ponto, foi realizada visita domiciliar pela equipe. Família formada por três membros, onde dois faziam uso abusivo de álcool e um sof ria violência doméstica. Realizou-se a estratificação de risco familiar aplicando a escala de Coelho e Savassi, cujo risco identificado foi máximo (escore 22). O ecomapa evidenciou vínculos intrafamiliares e comunitários frágeis. Considerações finais: $O$ uso das ferramentas proporcionou à equipe o reconhecimento sistemático e objetivo das fragilidades familiares e da rede social de apoio. Além disso, contribuiu para a identificação das necessidades de saúde da família, subsidiou um planejamento do cuidado adequado às suas vulnerabilidades, justificando a priorização das famílias e enriquecendo a qualidade da assistência prestada.

Palavras-chave: Estratégia saúde da família, Vulnerabilidade em saúde, Assistência integral à saúde, Relações interpessoais.

\begin{abstract}
Objective: To report the experience of the family health strategy team in the use of family approach tools to stratify the risk of vulnerability. Experience report: The selection of the family occurred in October 2020, and, from this point, one home visit was made by the team. A family of three members, where two were abusive to alcohol and one suffered domestic violence. Family risk stratification was performed by applying the Coelho and Savassi scale, whose identified risk was maximum (score 22). The ecomap showed fragile intraf amily and community bonds. Final considerations: The use of the tools provided the team with systematic and objective recognition of family weaknesses and the social support network. In addition, it contributed to the identification of family health needs, subsidized a planning of care appropriate to its vulnerabilities, justifying the prioritization of families and enriching the quality of care provided.
\end{abstract}

Key words: Family health strategy, Health vulnerability, Comprehensive health care, Interpersonal relations.

\footnotetext{
${ }^{1}$ Rede Nordeste de Formação em Saúde da Família (RENASF/UFMA), São Luís - MA.

*E-mail: elida.chaves@discente.ufma.br.

2 Universidade Federal do Maranhão (UFMA), São Luís - MA.

${ }^{3}$ Universidade de Porto, Concelho do Porto - Portugal.

${ }^{4}$ Hospital Sarah de Neurorreabilitação, São Luís - MA.
}

SUBMETIDO EM: 12/2021 


\begin{abstract}
RESUMEN
Objetivo: Relatar la experiencia del equipo de estrategia de salud de la familia en el uso de herramientas de enfoque familiar para estratificar el riesgo de la vulnerabilidad. Informe de experiencia: La selección de la familia se produjo en octubre de 2020, y, a partir de este momento, se realizó una visita domiciliada por parte del equipo. Una familia de tres miembros, donde dos abusaron del alcohol y uno sufrió violencia doméstica. La estratificación del riesgo familiar se realizó aplicando la escala de Coelho y Savassi, cuyo riesgo identificado fue máximo (puntuación 22). El ecomap mostró frágiles vínculos intrafamiliares y comunitarios. Consideraciones finales: El uso de las herramientas proporcionó al equipo el reconocimiento sistemático y objetivo de las debilidades familiares y de la red de apoyo social. Además, contribuyó a la identificación de las necesidades de salud de la familia y subvencionó una planificación de la atención adecuada a sus vulnerabilidades, justificando la priorización de las familias y enriqueciendo la calidad de la atención brindada.
\end{abstract}

Palabras clave: Estrategia de salud familiar, Vulnerabilidad en salud, Atención integral de salud, Relaciones interpersonales.

\title{
INTRODUÇÃO
}

O núcleo familiar é constituído por pessoas que, em suas singularidades, mantêm ligações e relações interdependentes. Portanto, quando alguém adoece ou acontece algo que modifica a dinâmica familiar, influência em cada membro individualmente ou no núcleo como um todo, resultando em demanda de reordenamento para enfrentar a nova situação, passando a necessitar de ações efetivas para a sua reestruturação (DIAS JF, et al., 2017).

O modelo da assistência centrado na família é considerado pelo Institute of Medicine como um dos seis requisitos para a alta qualidade no cuidado praticado pelos profissionais e serviços na saúde, porque permite a inserção da família e seus membros no planejamento das ações e nas tomadas de decisões, reconhecendo a família como parceira da equipe (SILVA TRG, et al., 2016).

Ao incluir a família como centro do cuidado, o profissional favorece o seu empoderamento, além de ser uma estratégia de estreitamento de vínculo (COATS H, et al., 2018). Há diminuição dos fatores estressores quando se inclui a família no cuidado, possibilita a avaliação da sua estrutura e funcionamento, bem como a identificação das necessidades de outros membros que possam precisar também de cuidados (LOR M, et al., 2016).

Os movimentos de reforma mais recentes e a partir da Conferência Internacional sobre Cuidados Primários de Saúde, Alma-Ata em 1978, têm destacado a importância da Atenção Primária à Saúde (APS) na organização dos sistemas de saúde e ordenadora do cuidado, para favorecer o acesso oportuno e adequado aos serviços e toda população adscrita de um território (ALMEIDA PF, et al., 2018).

Para que isso ocorra a Estratégia de Saúde da Família (ESF), desde 2006, proporciona uma mudança no processo de trabalho em saúde, que engloba o profissional, usuário, família e a co munidade. Os profissionais que atuam na ESF precisam realizar diagnóstico situacional da sua área, para que assim conheçam a realidade das famílias e das situações de risco que envolvem o território adscrito, na perspectiva de desenvolver ações, no âmbito individual e coletivo, de promoção, proteção e reabilitação da saúde, valorizando vínculo e acolhimento (LEITE MT, et al., 2016).

A fim de entender cada uma destas famílias e conhecer suas necessidades, as ferramentas de abordagem familiar e instrumentos que avaliam a vulnerabilidade, auxiliam os profissionais na identificação de fatores de risco que justifiquem a priorização do atendimento, sistematizando práticas, como a visita domiciliar, o diagnóstico situacional e a intervenção precoce (FRANÇA EPFB, et al., 2017).

O Ministério da Saúde (MS) recomenda a estratificação de risco para o conhecimento de diversas vulnerabilidades, entre elas a familiar. Para avaliar o grau de vulnerabilidade de uma família, há a Escala de Risco Familiar validada por Coelho e Savassi, que objetiva conhecer as famílias da área adscrita da ESF, possibilitando a reorganização do processo de trabalho da equipe, bem como o estabelecimento de vínculo entre as famílias e os profissionais (REGO AS, et al., 2016). 
Além da estratificação do grau de vulnerabilidade para o planejamento do cuidado, existem outras ferramentas (Genograma, Ecomapa, Ciclo de Vida Familiar, entre outras) que permitem conhecer a estrutura familiar, como sua composição, funções, papéis, formas de interação e organização entre os membros e o meio na qual está inserida (BRASIL, 2013).

O ecomapa consiste em uma imagem, construída por meio de símbolos, linhas e setas, onde os membros da família se localizam em um círculo central e fora dele existem pessoas, instituições, grupos que eles interagem ou não. Essa interação ou a ausência dela é de acordo com o desenho das linhas, que podem ser tracejadas, contínuas, com barras, indicando o tipo de conexão existente e o fluxo de energia (indicado pela direção da seta), resultando na visualização da rede de apoio da família (SIQUEIRA LD, et al., 2019).

Pensar em gestão ou na produção do cuidado é considerar um sistema que envolva vários aspectos de caráter individual e coletivo, com práticas e atitudes multiprofissionais que precisam ser, constantemente, repensadas, analisadas e até modificadas, dando sustentação à dinâmica do cuidado (SIEWERT JS, et al., 2017).

Os usuários querem solução e direcionamento para os seus problemas quando procuram uma unidade de saúde, para que isso ocorra, há necessidade de uma rede de atenção estruturada, com os serviços e profissionais que se comprometam com o cuidado, com foco na integralidade da assistência, sendo, portanto, a produção do cuidado o caminho para se chegar à cura, à promoção e proteção da saúde (LEITE MT, et al., 2016). O presente trabalho teve como objetivo relatar a experiência da equipe da ESF no uso de ferramentas de abordagem familiar para estratificação do risco de vulnerabilidade da família.

\section{RELATO DA EXPERIÊNCIA}

Trata-se de um relato da experiência da utilização de ferramentas de abordagem familiar e estratificação de risco de vulnerabilidade na ESF, que aconteceu em outubro de 2020 em São Luís, Maranhão.

A proposta consistiu na aplicação da ferramenta para identificação das necessidades de saúde de uma família residente e da sua relação com os profissionais e serviços de saúde da Unidade Básica de Saúde (UBS) na qual está inserida. A equipe faz parte de uma UBS localizada na periferia de São Luís, cuja área adscrita compreendia seis microáreas, abrangendo 12 ruas e 60 quadras, com aproximadamente 4.500 pessoas. A equipe era composta por uma enfermeira, uma técnica em enfermagem e quatro Agentes Comunitários de Saúde (ACS). No período em que a atividade foi desenvolvida, a equipe apresentava déficit de dois ACS e do profissional médico.

Cada ACS selecionou uma família vulnerável de sua área adscrita considerando os problemas já observados em visitas domiciliares da equipe. Posteriormente, realizou-se uma reunião entre a equipe da ESF, psicólogo e assistente social do Núcleo Ampliado de Saúde da Família (NASF) e os pesquisadores, onde cada ACS apresentou sua família a ser trabalhada. Selecionou-se então, consensualmente, a família mais vulnerável entre as apresentadas, que foi abordada no presente estudo.

Após decisão da equipe, foi realizada uma visita domiciliar para a coleta dos dados e aplicação das ferramentas de abordagem familiar. Por fim, realizou-se a estratificação do risco familiar aplicando a Escala de Risco Familiar de Coelho e Savassi, além da representação gráfica da família no Ecomapa.

Trata-se de uma família constituída por três pessoas (dois idosos e um adulto), que residem em uma microárea descoberta, porém a sua indicação foi feita por uma ACS que já havia atuado na microárea anos antes da permuta pra outro território. A casa é de alvenaria, sem revestimento, localizada em rua sem saneamento básico (não realizam nenhum tipo de tratamento da água para consumo). A casa possui quatro cômodos (quarto, sala, banheiro interno e cozinha). Embora o banheiro esteja localizado no interior da residência, não há sistema de descarga no vaso sanitário. O lixo circunda toda a casa, a céu aberto. $O$ interior da casa é cheio de entulhos e materiais recolhidos da rua. Há animais no interior da residência, sendo alimentados diretamente no chão, tornando o ambiente ainda mais insalubre. A renda familiar é de um salário mínimo. 
O casal de idosos vive em união estável. O terceiro adulto morador é fruto de outro relacionamento da idosa. O patriarca é aposentado, 66 anos, nega doenças preexistentes, deambula com dificuldade em decorrência de uma lesão no pé há mais de 20 anos, nega tabagismo e etilismo (fato contestado pela esposa, que afirma o uso abusivo). A mulher, pessoa índice do cuidado, possui 63 anos, hipertensa, afirma tabagismo e etilismo, relata sofrer violência doméstica. $O$ filho de 39 anos, desempregado, faz uso abusivo de álcool e outras drogas, não participa do cuidado da mãe.

Diante da anamnese realizada, notou-se que as histórias de vida que compõem a trajetória dessa família e a provável associação com um ambiente desprovido de adequadas condições sanitárias e de saúde concorrem para uma situação de fragilidade. Nessa perspectiva, o contexto adverso exige um cuidado coordenado e focado para que a família em questão tenha suas complexas necessidades sociais e de saúde acolhidas, com práticas direcionadas para os determinantes sociais da saúde, privilegiando a identificação precoce de agravos e fatores de riscos associados à situação de vulnerabilidade familiar.

Para responder aos desafios evidenciados, primeiramente, o contato com as ferramentas foi disponibilizado por meio de um processo interativo, no qual a enfermeira explanou para a equipe acerca dos instrumentos a serem utilizados para abordagem da família-alvo no ambiente de cuidados primários, nomeadamente a escala de Coelho e Savassi e o ecomapa.

De acordo com a escala, a soma resultou no score 23 , o que classifica a família com RISCO MÁXIMO (R3) de vulnerabilidade, apresentando pontuação nos seguintes critérios: deficiência física e mental (6), baixas condições de saneamento (3), desnutrição grave (3), drogadição (6), desemprego (2), analf abetismo (2), hipertensão arterial sistêmica (1). Pode-se observar o ecomapa da família em estudo na Figura 1:

Figura 1: Ecomapa da família, São Luís/MA, 2020.

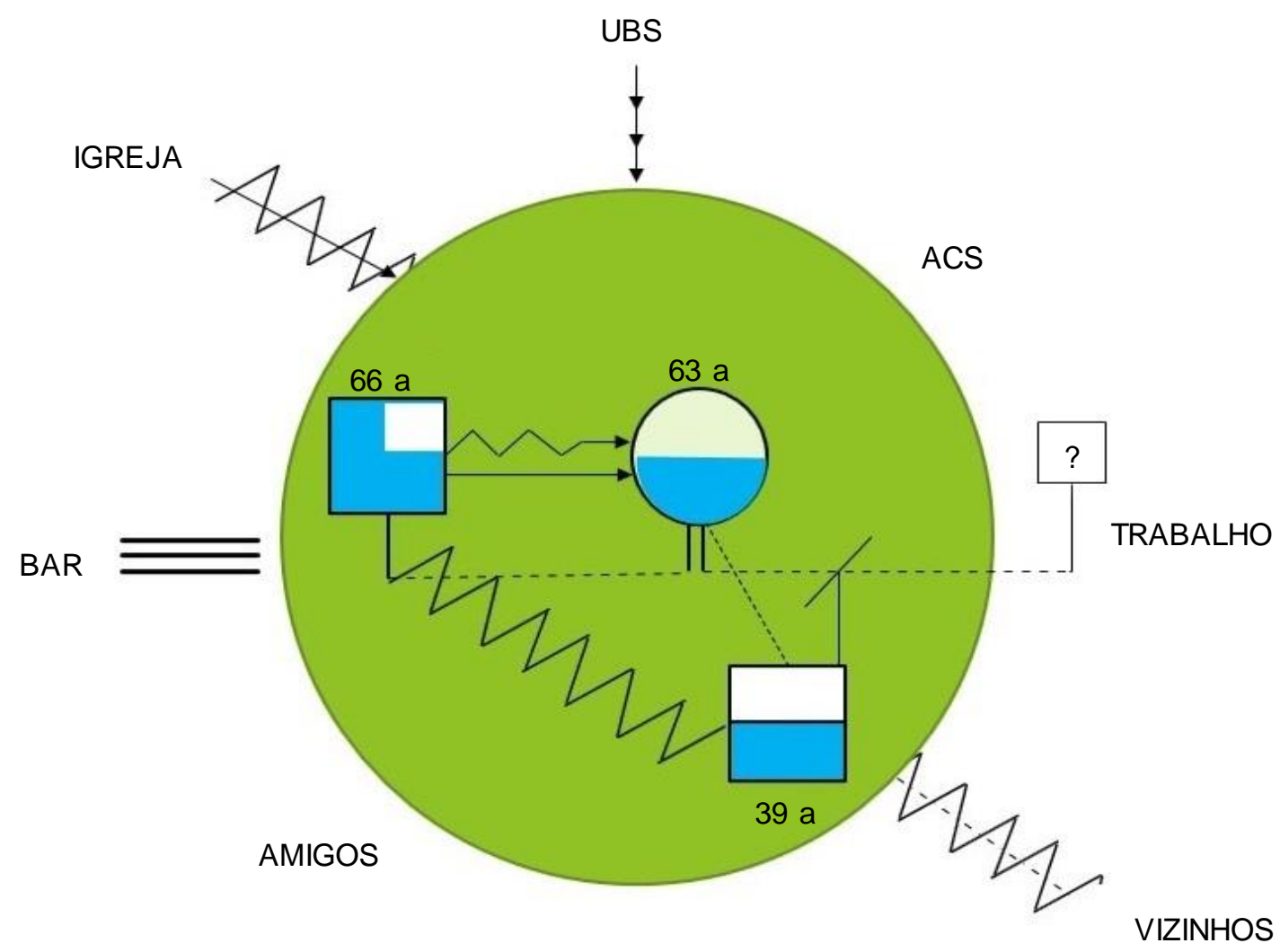

Fonte: Lima ECC, et al., 2022.

O tipo de conexão é representado pelas linhas que quando contínuas possuem forte ligação; linhas pontilhadas, ligação frágil; ausência de linhas quando não há qualquer conexão; linha sinuosa, significa relação estressante. As setas indicam o fluxo e a energia da relação (SOUZA IP, et al., 2016). 
No centro do ecomapa observa-se a dinâmica familiar, em que os sujeitos do sexo masculino são representados pela figura do quadrado e do sexo feminino pelo círculo. O primeiro quadrado que representa o marido está caracterizado com o quadrado menor no seu canto superior direito, indicando presença de transtorno mental. As cores que dividem as figuras representam o uso de drogas lícitas e ilícitas.

\section{DISCUSSÃO}

Para o relato da experiência proposta, é importante compreender que os profissionais de saúde da atenção primária podem usar abordagens distintas para compreensão de processos familiares e gerenciamento de suas interações com as famílias pelas quais são responsáveis. Nesta linha de pensamento, a aplicabilidade de ferramentas de abordagem familiar permite construir um conjunto de conhecimentos sobre a dinâmica e complexidade da estrutura familiar, além de ampliar a interatividade com a equipe de saúde.

\section{Escala de Estratificação de Risco Familiar}

O uso da Escala de Risco Familiar de Coelho e Savassi permitiu classificar a família segundo o risco biológico ou social fornecendo uma base instrumental para direcionar o processo de trabalho e práticas da equipe de saúde. Este instrumento utiliza doze critérios, utilizando as sentinelas de riscos da Ficha $A$ do Sistema de Informação da Atenção Básica (SIAB) em desuso, adaptada para a Ficha de Cadastro Domiciliar e Territorial (eSUS). Propõe uma pontuação para cada critério sentinela, podendo se repetir de acordo com o número de vezes que ela surgir na família e variar de 0-25 pontos (MOURA FMN, et al., 2016).

Desta forma, refere-se a uma forma objetiva de avaliar cada núcleo familiar, utilizando dados de fácil acesso a toda equipe, permitindo, assim, identificar as vulnerabilidades familiares, o conhecimento das situações mais urgentes de atuação da equipe de ESF e o direcionamento das abordagens e intervenções mais prioritárias (MONTEIRO WF, et al., 2021).

Observou-se que com preparação e capacitação prévia quanto ao manuseio e seleção dos critérios pela equipe, a ferramenta mostrou-se de fácil aplicabilidade e eficiente na avaliação do risco familiar, facilitando assim a atuação da equipe.

Outros estudos também demonstraram bons resultados com o uso do instrumento, com melhoria do trabalho em equipe, fortalecimento da interdisciplinaridade e a possibilidade de avaliação in loco dos diversos perfis populacionais presentes na área de abrangência da ESF (MOURA FMN, et al., 2016; MONTEIRO WF, et al., 2021).

\section{Ecomapa}

Após a estratificação do risco familiar, foi elaborado o ecomapa, que se trata a uma representação gráfica, dinâmica, que permite a observação do contexto social, cultural e econômico em que a família se insere, facilitando a observação dos aspectos que se relacionam com processo de saúde / doença e seus determinantes, formulando uma visão ampliada sobre a família (NASCIMENTO LC, et al., 2014).

A partir do conhecimento da representação de determinado momento desta família, a equipe de saúde da ESF pode fomentar estratégias de intervenções em saúde, com cuidado centrado à família e que possam auxiliar na resolução e enfrentamento dos problemas encontrados, respeitando os princípios de integralidade e equidade para esse núcleo familiar (BARBOSA NG, et al., 2021).

Referente aos vínculos interpessoais intrafamiliares, observa-se que o esposo possui uma relação dominante e conf lituosa com a esposa e uma relação estressante com o filho dela. A ligação entre mãe e filho é frágil, sendo que o filho não mantém contato com o pai biológico. Pode-se inferir que se trata de uma família vulnerável psicossocialmente, sendo que a violência intraf amiliar muitas vezes faz parte de uma manif estação da desigualdade social e está diretamente relacionada às relações de poder (BRESSAN CR e LOCH HS, 2018).

Com relação aos vínculos da família com os demais sistemas, observa-se que a família não possui mecanismos de apoio comunitário além da UBS, sendo apenas no sentido unidirecional, pois os indivíduos 
da família não se co-responsabilizam pelo cuidado. Outra relação frágil e conflituosa refere-se a representantes da igreja, que ao tentarem exercerem seu papel assistencial, frequentemente são expulsos do domicílio. O ecomapa permitiu visualizar também um vínculo fraco da família com demais membros da comunidade, representando uma relação tumultuosa com os vizinhos. Poucas conexões da família com o meio na qual está inserida, ou até mesmo entre seus componentes, exigem dos profissionais maior atenção no momento de priorizar estratégias (SOUZA IP, et al., 2016).

Observa-se uma relação muito forte com estabelecimentos onde há consumo frequente de bebidas alcoólicas, fato que gera outros problemas e conflitos interfamiliares. O consumo excessivo de álcool pode ser um indicador precoce de vulnerabilidade familiar, que af eta a qualidade das relações e um fator negativo para resultados favoráveis em saúde (GOWIN JL, et al., 2017).

Além disso, há presença de doenças mentais no núcleo familiar, que também devem ser foco de atenção da ESF, é fundamental o apoio da comunidade. A implementação de terapias de suporte à família é necessária para a compreensão do fenômeno do adoecimento, desmistificando a doença e as suas formas de tratamento (WEBER CAT e JURUENA MF, 2017).

Com observação e análise das relações e demandas apresentadas pela família, a equipe da ESF pode direcionar a abordagem e ferramentas efetivas, propondo intervenções e atuando sobre as condições vulneráveis deste núcleo familiar, bem como as relações entre seus membros e os sistemas externos. A implementação das ferramentas de abordagem familiar pode promover a prestação de cuidados voltados para qualificar o atendimento e melhorar a experiência da família por meio de um plano de cuidados coordenado e integrado, cujo alvo é a promoção e proteção da saúde deste núcleo familiar.

O uso de ferramentas para abordagem de famílias vulneráveis requer processos de trabalho bem estruturados e o envolvimento de uma equipe multiprofissional. No entanto, devido a questões multifatoriais que dificultam a fluidez do trabalho no contexto da ESF, muitas vezes as equipes não estão preparadas para o uso desse tipo de estratégia. A utilização dessas ferramentas fundamenta as ações da equipe, facilita a visão sob a família e a sua rede de apoio, contribui para o estabelecimento de parcerias que possam colaborar no processo de cuidar dos envolvidos, além de favorecer o raciocínio clínico e o conhecimento científico, justificando a priorização das famílias e enriquecendo a qualidade da assistência prestada.

\section{REFERÊNCIAS}

1. ALMEIDA PF, et al. Coordenação do cuidado e Atenção Primária à Saúde no Sistema Único de Saúde. Saúde Debate, 2018; 42(número especial):244-260.

2. BARBOSA NG, et al. Genograma e ecomapa como estratégias lúdicas de ensino de enfermagem na Atenção Primária à Saúde. Revista Brasileira de Enfermagem, 2021;74.

3. BRASIL. Caderno de atenção 2013.2 Disponível em: https://bvsms.saude.gov.br/bvs/publicacoes/caderno_atencao_domiciliar_melhor_casa.pdf. Acessado em: 3 de Dezembro de 2020.

4. BRESSAN CR, LOCH HS. Violência Intrafamiliar e em especial a Violência Sexual Intrafamiliar Contra Crianças e Adolescentes: do espaço privado ao espaço da política pública. Sociedade em Debate, 2018;24(2):185-206.

5. COATS H, et al. Nurses' Reflections on Benefits and Challenges of Implementing Family-Centered Care in Pediatric Intensive Care Units. Am. J. Crit. Care, 2018;27(1):52-58.

6. COELHO FLG, SAVASSI LCM. Aplicação de Escala de Risco Familiar como instrumento de priorização das Visitas Domiciliares. Rev. Bras. Med. Fam. Comunidade, 2004;1(2):19-26.

7. DIAS JF, et al. Atenção domiciliar no âmbito da reabilitação e prática centrada na família: aproximando teorias para potencializar resultados. Rev. Ter. Ocup. Univ., 2017;28(2):206-13.

8. FRANÇA PFBP, et al. Identificação e Classificação de Risco Familiar em uma comunidade assistida por uma Unidade de Saúde da Família em Recife-Pe. Revista Brasileira de Inovação Tecnológica em Saúde, 2017;7(3).

9. GOWIN JL, et al. Vulnerability for Alcohol Use Disorder and Rate of Alcohol Consumption. Am. J. Psychiatry, 2017; 1;174(11):1094-1101.

10. LEITE MT, et al. Gestão do cuidado na estratégia saúde da família: revisão narrativa. Rev. Aten. Saúde, 2016; 14(48):106-115. 
11. LOR M, et al. A proposed model of person, family, and culture centered nursing care. Nursing Outlook, 2016; 64(4):352-366.

12. MONTEIRO WF, et al. Genograma e ecomapa: contribuições da enfermagem brasileira. Texto Contexto Enferm., 2014;23(1):211-20.

13. MOURA FMN, et al. Aplicação da escala de risco familiar na atenção básica. Extensão em Ação, 2016; 1(10).

14. NASCIMENTO LC, et al. Genogram and ecomap: brazilian nursing contributions. Texto \& Contexto, 2014;23(1):211220.

15. REGO AS, et al. Estratificação de risco familiar no contexto da estratégia de saúde da família. Ver. Enferm. UFPE, 2016;10(3):977-84.

16. SIEWERT JS, et al. Gestão do cuidado integral em enfermagem: reflexões sob a perspectiva do pensamento complexo. Rev, Min. Enferm. (REME), 2017;21e-1047.

17. SILVA TRG, et al. Cuidado centrado na família na perspectiva de enfermeiras da Unidade de Terapia Intensiva Neonatal. Ver. Rede de Enfermagem do Nordeste (Rene), 2016;17(5):643-50.

18. SIQUEIRA LD, et al. Influence of the social context in smoking during pregnancy. Rev. Bras. Enferm., 2019;72(suppl 3):259-265.

19. SOUZA ÍP, et al. Genograma e Ecomapa como ferramentas para compreensão do cuidado familiar no adoecimento crônico de jovem. Texto Contexto Enferm., 2016;25(4).

20. WEBER CAT, JURUENA MF. Paradigmas de atenção e estigma da doença mental na reforma psiquiátrica brasileira. Psicologia, Saúde e Doenças, 2017; 18(3), 640-656. 